\title{
COVID-19 e Segurança Alimentar: a crise dentro da crise ${ }^{1}$
}

\author{
COVID-19 and Food Security: the crisis within the crisis
}

COVID-19 y Seguridad Alimentaria: la crisis dentro de la crisis

Matilde de Souza ${ }^{2}$

Jéssica Rúbia Gonçalves ${ }^{3}$

Lauana Pereira Domingos Alves ${ }^{4}$

Bárbara Luana Pereira Pacheco ${ }^{5}$

DOI: $10.5752 / \mathrm{P} .1809-6182.2020 \mathrm{v} 17 \mathrm{n} 3 \mathrm{p} 2$

Recebido em: 13 de maio 2020 Aceito em: 25 de agosto de 2020

\begin{abstract}
Resumo
Este artigo trata da segurança alimentar no contexto da pandemia da Covid-19 e discute a garantia de que acesso ao alimento integra ações para salvar vidas. Salienta-se que tais ações não desconsideram os desafios da adoção de medidas de contenção do avanço da doença.

Palavras-chave: Segurança alimentar. Covid-19. Crise alimentar.
\end{abstract}

\begin{abstract}
This article deals with food security in the context of the Covid-19 pandemic and discusses the guarantee that access to food integrates actions to save lives. We emphasize that such initiatives do not disregard the challenges of adopting measures to contain the progress of the disease.

Keywords: Food Security. Covid-19. Food crisis.

\section{Resumen}

Este artículo trata de la seguridad alimentaria en el contexto de la pandemia de Covid-19 y analiza la garantía de que el acceso a los alimentos integra acciones para salvar vidas. Cabe señalar que tales acciones no ignoran los retos de adoptar medidas para contener la propagación de la enfermedad.

Palabras-clave: Seguridad Alimentaria. Covid-19. Crisis alimentaria.
\end{abstract}

1 O presente artigo foi realizado com apoio da Coordenação de Aperfeiçoamento de Pessoal de Nível Superior - (Brasil) CAPES Código de Financiamento 001. Projeto CNPQ 405013/2016-7. As autoras agradecem a Fundação de Amparo à Pesquisa do Estado de Minas Gerais - FAPEMIG pelo apoio financeiro no Projeto APQ-01085-16: Mudanças Climáticas e Segurança Hídrica: análise do papel da Agência Nacional de Águas na internalização de políticas internacionais de mitigação e adaptação dos efeitos das mudanças climáticas ao modelo brasileiro de governança de recursos hídricos (Chamada 01/2016 - Demanda Universal).

2 Professora do Departamento de Relações Internacionais da Pontifícia Universidade Católica de Minas Gerais (PUC Minas). Doutora em Ciências Humanas: Sociologia e Política (UFMG). Belo Horizonte, Minas Gerais, Brasil. ORCID ID: 0000-0001-5241-5644.

3 Mestra em Relações Internacionais pela Pontifícia Universidade Católica de Minas Gerais (PUC Minas). Belo Horizonte, Minas Gerais, Brasil. ORCID ID: 0000-0001-5957-8331.

4 Graduanda em Relações Internacionais pela Pontifícia Universidade Católica de Minas Gerais (PUC Minas). Belo Horizonte, Minas Gerais, Brasil. ORCID ID: 0000-0003-4729-8919.

5 Graduanda em Relações Internacionais pela Pontifícia Universidade Católica de Minas Gerais (PUC Minas). Belo Horizonte, Minas Gerais, Brasil. ORCID ID: 0000-0002-8806-1224. 


\section{Introdução}

Uma das questões que tem suscitado preocupações face aos desafios enfrentados pela humanidade em decorrência da pandemia da COVID-19 é a ameaça da fome. Esse tema não é novo na agenda da política internacional e muitos esforços têm sido empreendidos no sentido de resolvê-lo através de políticas que forneçam condições para garantir maior segurança alimentar. Contudo, em situações de calamidade, como a atual, o problema ressurge e há riscos de que se perca parte dos efeitos positivos de ações internacionais e de governos de vários países no sentido de reduzir o número de pessoas que não têm acesso a comida.

Em entrevista concedida ao jornal O Estado de São Paulo, Daniel Balaban, responsável pelo escritório brasileiro do Programa Mundial de Alimentos, da Organização das Nações Unidas (ONU), alerta para o risco de que, em decorrência da pandemia, 130 milhões de pessoas se somarão aos atuais 135 milhões que se encontram em situação de fome crônica no mundo (BALABAN, 2020). O mapa que ilustra o texto da entrevista informa as regiões mais afetadas pela insegurança alimentar no período de 2016 a 2018 e expõe vulnerabilidades importantes em diversos países do Sul Global. A entrevista alerta para o risco de que camadas da população que haviam saído da situação de fome crônica não consigam se manter nesse patamar, inclusive no Brasil. Tal situação pode ocorrer, seja por debilidades de ações dos governos, seja por restrições de acesso às pessoas que precisam receber doações ou ainda por dificuldades de manter a produção de alimentos e garantir preços acessíveis à população de baixa renda, ou até a diminuição da renda mensal das famílias.
Há receio de uma crise mundial de fome e instabilidade, conforme opinião de David M. Beasley, Diretor Executivo do mesmo Programa da ONU acima citado. Para ele, a pandemia ameaça detonar uma crise humanitária global sem precedentes, dado que os desafios são muitos e envolvem cortes de financiamento a ações do Programa, fechamento de fronteiras, dificuldades de acesso às pessoas que necessitam socorro e de manutenção dos fluxos de mercadorias e de pessoas que prestam ajuda humanitária (BEASLEY, 2020).

Os líderes do Programa Mundial de Alimentos citados acima - Daniel Balaban e David M. Beasley - não questionam medidas preventivas, como o isolamento social, para evitar a ampliação do contágio do coronavírus. Porém, alertam para possíveis consequências da pandemia no que se refere ao agravamento da fome no mundo, caso não sejam adotadas medidas que assegurem aos mais pobres acesso aos alimentos e também que não impeçam ou dificultem a oferta de ajuda humanitária nas regiões onde são mais necessárias e urgentes. A pandemia da COVID-19 apresenta à humanidade o desafio de salvar vidas pela restrição do contato social e medidas de isolamento, com inegáveis impactos na economia, concomitantemente ao desafio de salvar vidas pela preservação das condições de sobrevivência das populações mais pobres do mundo, que demandam ações também com impactos na economia.

Nesse sentido, a América Latina e o Caribe poderão ter a segurança alimentar seriamente prejudicada devido aos efeitos da pandemia. No mais recente relatório da Organização das Nações Unidas para Alimentação e Agricultura (FAO) sobre segurança alimentar (FAO et al., 2020), projeta-se que a região não vai conseguir alcançar o Objetivo do Desenvolvimento 
Sustentável 2 (ODS2), cuja meta é, dentre outras, acabar com a fome até 2030. Essa projeção parte de um contexto já desfavorável para o combate à fome, que vem sendo enfrentado nos últimos 5 anos. Em 2019, na América Latina e Caribe, cerca de 47,7 milhões de pessoas passavam fome. Estima-se que em 2030 esse número subirá para quase 67 milhões de pessoas, isso sem contar os impactos causados por essa Pandemia (FAO et al., 2020).

Entende-se que são interligados os desafios de conter a pandemia e evitar a fome, garantindo a segurança alimentar, e que ambos devem ser enfrentados simultaneamente. Porém, este artigo enfatiza a discussão da segurança alimentar no contexto da crise sanitária desencadeada pela pandemia.

\section{A Crise Sanitária Entrelaçada à Crise Alimentar}

A situação da segurança alimentar tem se deteriorado nas últimas décadas. Segundo dados da FAO (2019), atualmente mais de 820 milhões de pessoas sofrem de fome crônica no mundo, ao passo que também verifica-se tendência ao aumento do sobrepeso e da obesidade. Para a FAO (1996), "a segurança alimentar existe quando todas as pessoas têm, permanentemente, acesso físico e econômico a alimentos seguros, nutritivos e suficientes, que atendam às suas necessidades dietéticas e preferências alimentares para uma vida ativa e saudável." (FAO, 1996, tradução nossa) ${ }^{6}$.

Desse modo, a segurança alimentar implica 4 dimensões: a) Disponibilidade: trata da neces-

\footnotetext{
6 Food security exists when all people, at all times, have physical and economic access to sufficient, safe and nutritious food to meet their dietary needs and food preferences for an active and healthy life.
}

sidade de manter oferta de alimentos suficientes para a população; b) Acesso: refere-se à garantia de acesso tanto físico quanto econômico aos alimentos; c) Utilização: relaciona-se à segurança dos alimentos para o consumo, seja em termos de higiene ou em sua qualidade nutricional; d) Estabilidade: dimensão temporal do conceito de segurança alimentar e que se refere à ideia de acesso permanente aos alimentos, reforçando a necessidade de sua disponibilidade regular. $\mathrm{O}$ quadro 1, abaixo, apresenta algumas variáveis que compõem as dimensões do conceito.

Quadro 1- Dimensões do Conceito de Segurança Alimentar

\begin{tabular}{|c|l|l|l|}
\hline Disponibilidade & \multicolumn{1}{|c|}{ Acesso } & \multicolumn{1}{c|}{ Utilização } & Estabilidade \\
\hline & $\begin{array}{l}\text { Mercado, de- } \\
\text { manda, preço, } \\
\text { poder de com- } \\
\text { pra, questões } \\
\text { socioeconô- } \\
\text { estoquecão, oferta, comércio, } \\
\text { micas. }\end{array}$ & $\begin{array}{l}\text { Aualidade da } \\
\text { água, hábitos } \\
\text { alimentares, } \\
\text { qualidade dos } \\
\text { alimentos. }\end{array}$ & $\begin{array}{l}\text { dimensês } \\
\text { anteriores se } \\
\text { realizarem } \\
\text { conjunta- } \\
\text { mente. }\end{array}$ \\
\hline
\end{tabular}

Fonte: Elaboração própria, com base em: FAO, 1996; FAO, 2008.

Considerando-se o conceito de segurança alimentar e providências para garantir acesso ao alimento para todos, é possível perceber o quão desafiador é erradicar a fome e garantir a segurança alimentar no atual cenário. Esses desafios tendem a se intensificar nesse contexto da pandemia da COVID-19, podendo gerar crise alimentar, como já aludido na introdução deste artigo. A pandemia vem afetando a demanda por alimentos, ligada à dimensão acesso, e também a sua oferta, ligada à dimensão disponibilidade. Algumas medidas como quarentenas, fechamentos de fronteiras e do comércio levam a que o consumidor compre mais do que normalmente faria, visando estocar alimentos, e esse comportamento pode afetar fortemente a oferta, gerando aumento da demanda por alimentos muito acima da média (FAO, 2020b).

O Relatório Global de Crises Alimentares (FSIN, 2020) aponta que a COVID-19, con- 
juntamente com outros fatores como choques econômicos e eventos climáticos extremos, será um dos principais impulsionadores do agravamento da insegurança alimentar ${ }^{7} \mathrm{em} 2020$. Além disso, projeta que o número de pessoas em situação de insegurança alimentar pode dobrar em consequência da pandemia. Por essa razão, a FAO estima que o impacto da pandemia na insegurança alimentar será, provavelmente, o aumento do já elevado número de pessoas atualmente nessa situação, e a insegurança alimentar poderá atingir cerca de 265 milhões de pessoas até o fim deste ano (FSIN, 2020).

Acesso a víveres é uma das dimensões mais seriamente afetadas nas condições da atual crise sanitária. Isso porque, além da pandemia da COVID-19 e em decorrência dela, verifica-se dramática combinação entre aumento dos preços, com redução da disponibilidade dos alimentos, e aumento do desemprego, com redução do poder de compra, todos fatores que comprometem seriamente o acesso aos alimentos.

No caso da epidemia do Ebola, ocorrida em 2014 (FAO, 2014a), houve escassez de comida e impacto na segurança alimentar devido, principalmente, à interrupção do fluxo comercial de commodities alimentares. Outra consequência importante é o aprofundamento de vulnerabilidades já existentes:

O impacto na segurança alimentar pode levar famílias vulneráveis a recorrer a estratégias negativas de enfrentamento, que terão efeitos duradouros em suas vidas e meios de subsistência, incluindo redução no número de refeições, aumento da taxa de abandono escolar, meios reduzidos para cobrir gastos com saúde,

\footnotetext{
7 A FAO define insegurança alimentar como "ingestão insuficiente de alimentos devido à impossibilidade de os adquirir, seja por falta de abastecimento dos mercados locais, por falta de capacidade para os comprar ou produzir, ou por ambas as circunstâncias simultaneamente.” (FAO, 2014b, p. 6).
}

violência de gênero, venda de ativos produtivos, etc (FAO, 2020a, p. 4, tradução nossa). ${ }^{8}$

Importante notar que o preço dos alimentos é uma questão também ligada à dimensão acesso, por se tratar de uma variável que sofre muita flutuação em momentos de crise, seja pelo aumento do preço ao consumidor final ou pela redução do valor das commodities alimentares devido à contração da demanda. Segundo o índice de preços dos alimentos divulgado pela FAO, em março houve queda de 7.8 pontos em comparação com o mês de fevereiro. As maiores quedas foram observadas no açúcar (40,1 pontos) e nos óleos vegetais (19 pontos). No que diz respeito ao açúcar, medidas de isolamento social têm reduzido a demanda por retração do consumo; além disso, a baixa no preço dos combustíveis estimula a produção de açúcar ao invés de etanol (FAO, 2020c).

Há, ainda, um choque na oferta em termos de logística de alimentos, registrando-se redução na força de trabalho em países africanos, por exemplo, com possível repercussão em áreas como a agricultura, setores de produção e processamento de alimentos. Com as medidas de quarentena adotadas pelos países, há restrições nos transportes, o que pode impedir o acesso dos agricultores a insumos, produtos e mercados, além do risco de maior perda de alimentos e desperdício nas cadeias de suprimentos (CULLEN, 2020a; 2020b). A África é um continente que inspira especial preocupação pelo fato de que muitos países já afetados por crises alimentares lá estão localizados, porém a

\footnotetext{
which will have lasting effects on their lives and livelihoods, including re- duction in number of meals, increased school dropout rate, decreased means to cover health expenditures, gender-based violence, selling of productive assets, etc.
} 
América Latina e o Caribe possuem, também, alguns países que suscitam preocupação.

Em tentativas de encontrar condições para garantir segurança alimentar durante a pandemia, a FAO busca reorganizar sua ação humanitária, principalmente voltada para as regiões mais necessitadas. Tais condições envolvem iniciativas de apoio a pequenos produtores, distribuição de insumos agrícolas para as comunidades onde prevalecem a desnutrição e a pobreza, e medidas para estabilizar acesso aos alimentos e apoio do poder aquisitivo da população, inclusive com transferência de dinheiro para os mais necessitados, no intuito de garantir o atendimento de necessidades básicas das famílias e evitar empobrecimento ainda maior (FAO, 2020f ). É imprescindível o envolvimento dos tomadores de decisão, cujas ações são fundamentais para garantir o funcionamento dos sistemas alimentares. Desse modo, a FAO tem analisado como a pandemia afeta o setor de alimentação e a agricultura; tem produzido informes técnicos sobre a repercussão da crise sanitária nas condições necessárias à garantia de maior segurança alimentar, sistematiza o aprendizado com crises passadas e oferece síntese de decisões tomadas por Estados membros para mitigar efeitos da crise nos sistemas alimentares e agrícolas.

\section{América Latina e África: desafios da crise alimentar no contexto da pandemia}

Dados atuais da pandemia na América do Sul indicam que a COVID-19 soma cerca de 3.748 .800 pessoas afetadas, com cerca de 134 mil mortes (WORLDOMETERS, 20-?) ${ }^{9}$.

9 Esse Site atualiza diariamente os dados da pandemia para todos os países do globo. Os números aqui citados são referentes a 27/07/2020. Para maiores informações, consultar: https://www.worldometers.info/coronavirus/
O Brasil reúne cerca de $1 / 3$ do contingente de pessoas afetadas pela doença, seguido por Peru, Chile e Colômbia. O Equador, que foi palco de gravíssima crise e colapso do sistema de saúde no mês de abril, encontra-se atualmente na sexta posição. A situação é alarmante e o número de novos casos e de mortes continua aumentando. O cenário é de grande incerteza, por falta de maior conhecimento sobre a doença, ausência de medicamentos mais eficazes ou de uma vacina, e também por falta de dados e informações a respeito da evolução da pandemia e que poderiam ajudar a evitar danos maiores às pessoas e à economia.

Para a América Latina e Caribe (ALC), a FAO reconhece melhoras significativas na segurança alimentar, com redução da fome e desnutrição durante o final do século XX e primeira década do séc. XXI. Essa condição se deveu, segundo avaliação da Organização, a compromissos da sociedade e a implementação de políticas para enfrentamento do problema. Porém, mudanças políticas, econômicas e sociais mais recentes têm reduzido o alcance de medidas anteriormente tomadas. Desse modo, o número de pessoas subalimentadas voltou a aumentar, chegando, em 2017, a 39,3 milhões na ALC, o que representa $6,1 \%$ da população. Da mesma forma, aumentou a insegurança alimentar grave (FAO, 2018). Nessa situação, os grupos mais vulneráveis são as populações rurais em geral, mulheres e famílias de baixa renda e povos indígenas, considerando que a FAO avalia que as políticas implementadas não foram suficientes para melhorar as condições desses grupos, e medidas tomadas por governos mais recentemente eleitos têm piorado as condições de enfrentamento da fome (BALABAN, 2020).

As respostas à insegurança alimentar já vinham sofrendo revezes em razão de obstáculos 
ao impulsionamento do desenvolvimento e tal situação se agrava no atual cenário. São muitas as consequências para a ALC, segundo informe da Comissão Econômica para a América Latina e o Caribe (CEPAL), dentre elas a queda de preços de produtos primários, interrupção de cadeias globais de valor, redução da atividade econômica em geral (CEPAL, 2020a). Esses fatores repercutem no comércio internacional e provocam impactos sociais, dentre os quais se destacam a debilidade dos sistemas de saúde, a vulnerabilidade social de parte importante da população e a dependência do funcionamento das escolas para a garantia de acesso das crianças mais pobres a alimentação adequada.

Face ao conjunto de efeitos adversos, os países da região se reuniram em Santiago, no início do último mês de março, para discutir a coordenação de ações, tendo em vista garantir o funcionamento regular do sistema alimentar durante a crise da COVID-19. O compromisso diz respeito à garantia de "abastecimento de alimentos suficientes, inócuos e nutritivos para os 620 milhões de habitantes da região durante a pandemia da COVID-19." (FAO, 2020d). Nesse documento, os ministros e secretários de agricultura, pecuária, pesca, alimentação e desenvolvimento rural dos vários países da ALC afirmam a normalidade da produção de alimentos e que, face à possibilidade de maior pressão ao setor por prolongamento da crise sanitária, todos os países buscarão manter as cadeias locais, regionais e globais de abastecimento, com atuação coordenada, tendo em vista um conjunto de medidas que estão detalhadas no documento em referência.

Não obstante, a CEPAL (2020b) reconhece que a precarização do trabalho, o aumento do desemprego e a redução dos salários terão efeitos diretos no aumento das desigualdades na região e a projeção de redução do produto interno bruto (PIB) dos países poderá ter repercussão nas condições de atendimento às necessidades básicas da população mais pobre. Calcula-se queda de 5,3\% em média no PIB dos países, com um aumento de 4,4\% da população pobre da região, chegando a 29 milhões de pessoas. Por outro lado, projeta-se que a pobreza extrema aumentaria em torno de 2,9\%, atingindo cerca de 16 milhões de pessoas. Mesmo com essas projeções, a CEPAL reforça a necessidade das medidas de isolamento social, embora indique alternativas para a redução de danos às economias locais.

O continente africano possui atualmente ${ }^{10}$ aproximadamente 853.900 pessoas acometidas pela COVID-19 e cerca de 17.850 mortes. Os países com maior número de casos são África do Sul, Egito, Nigéria, Gana e Argélia, sendo que Sudão e Camarões aparecem dentre os seis países com o maior número de mortes (WORLDOMETERS, 20-?). Medidas de contenção da pandemia estão sendo tomadas e, em geral, seguem orientações da Organização Mundial de Saúde.

Salienta-se que, do total das pessoas que sofrem de insegurança alimentar no mundo, mais da metade vive em países africanos (FSIN, 2020). Alguns desses países são os mais seriamente acometidos pela insegurança alimentar, principalmente Etiópia, Nigéria, Moçambique, República Democrática do Congo e Sudão do Sul. Esses países são altamente dependentes da exportação de commodities e são afetados por choques de preços, secas, mudanças climáticas, alto índice de pobreza e deslocamentos internos, que potencializam a insegurança alimentar e tor-

10 Dados de 27/07/2020 - https://www.worldometers.info/ coronavirus/ 
na mais difícil sua superação (FAO, 2019). Esses países, tidos como mais vulneráveis, têm maior propensão a piorar a insegurança alimentar:

Embora a COVID-19 não discrimine, os 55 países e territórios que abrigam 135 milhões de pessoas com insegurança alimentar aguda e que necessitam de assistência humanitária, alimentar e nutricional são também os mais vulneráveis às consequiências dessa pandemia, uma vez que têm pouca ou nenhuma capacidade para lidar com os aspectos de saúde e socioeconômicos provocados pelo choque (FSIN, 2020, p. 4, tradução nossa). ${ }^{11}$

Esses países mais vulneráveis têm um quadro extremamente complexo para enfrentar: salvar os meios de subsistência e salvar vidas. A FAO argumenta que, ainda que vidas possam ser salvas do coronavírus, elas podem ser perdidas para a fome (FSIN, 2020). Isso porque medidas de contenção do avanço da doença devem preservar as oportunidades de acesso das pessoas a condições básicas de sobrevivência. Considerando esse cenário, em meados de abril os ministros da agricultura dos países que compõem a União Africana ${ }^{12}$ realizaram uma reunião virtual para discutir o impacto da $\mathrm{CO}$ VID-19 na região, tratando, principalmente, da questão de segurança alimentar. $\mathrm{O}$ evento foi organizado juntamente com a FAO e nele os ministros demonstraram profunda preocupação, já que a doença tem afetado não somente

11 While COVID-19 does not discriminate, the 55 countries and territories that are home to 135 million acutely food-insecure people in need of urgent humanitarian food and nutrition assistance are the most vulnerable to the consequences of this pandemic as they have very limited or no capacity to cope with either the health or socioeconomic aspects of the shock.

12 “A União Africana (UA) foi fundada em 2002, ocupando o lugar da antiga Organização da Unidade Africana, esta última atuante desde 1963. Com objetivo de prevenir conflitos na região e, ao mesmo tempo, promover o desenvolvimento do continente, a UA busca principalmente acelerar o processo de integração dos países africanos." (CEBRAFRICA, 2020). a saúde e a alimentação mas também dinâmicas sociais e econômicas da região (ONU NEWS, 2020). Sabe-se que esse problema não é exclusivo do continente africano, mas a situação das economias locais e o contingente de pessoas em condições de insegurança alimentar desafia fortemente a capacidade de respostas aos desafios de salvar vidas na região, no atual cenário.

Como tem ocorrido na grande maioria dos países, medidas de isolamento adotadas pelos países africanos repercutem na economia por meio da perda de produtividade e da queda no comércio. Além disso, é crescente o número de comunidades atingidas pela COVID-19, o que torna urgente a busca por iniciativas que atenuem o impacto das interrupções sobre o sistema de agricultura e de alimentação (ONU NEWS, 2020). Esses sistemas devem ser fortemente atingidos devido à dependência de mão de obra e à ausência de trabalhadores, podendo colocar em risco as plantações e as colheitas:
A pandemia pode devastar os meios de subsis- tência e a segurança alimentar, especialmente em contextos frágeis e particularmente para as pessoas mais vulneráveis que trabalham nos setores agrícolas e não-agrícolas infor- mais. Uma recessão global perturbará prin- cipalmente as cadeias de suprimento de ali- mentos (FSIN, 2020, p. 3, tradução nossa) ${ }^{13}$.

Desse modo, as populações mais pobres devem ser as mais atingidas pelas consequências da pandemia, e os já altos níveis de fome, pobreza e má nutrição tendem a se agravar. De acordo com a União Africana, é hora de os governos priorizarem a saúde e a segurança alimentar da população, levando em conta os

in the informal agri- cultural and nonagricultural sectors. A global recession will majorly disrupt food supply chains. 
diferentes contextos econômicos de cada região ou país, além de manterem o compromisso de erradicar a fome no continente até 2025 (ONU NEWS, 2020).

Apesar da dificuldade em prever desdobramentos da pandemia de COVID-19, a FAO tem se apoiado na experiência de situações anteriores similares. $\mathrm{O}$ que se consegue adiantar é que, nessas situações, alguns países já frágeis e com população vulnerável são ainda mais afetados pelas consequências das crises alimentares (FAO, 2020a). A epidemia do Ebola na África Ocidental, em 2014, por exemplo, demonstrou que, além da importância de aumentar as intervenções de ajuda humanitária para a garantia da segurança alimentar para as populações vulneráveis, é necessário esforço conjunto de vários setores, e que deve envolver principalmente o setor da saúde, para evitar a propagação do vírus, mas, ao mesmo tempo, evitar grande restrição na circulação e comercialização de alimentos (FAO, 2020e).

Experiências durante a crise dos preços entre 2007-2008 e em 2014, no surto do Ebola, demonstram a necessidade de agir com rapidez para minimizar os impactos:

“[...] desenvolvendo medidas políticas apropriadas, mantendo e aprimorando as intervenções humanitárias de segurança alimentar e protegendo os meios de subsistência e o acesso a alimentos das pessoas mais vulneráveis, particularmente aquelas em contextos de crise alimentares." (FAO, 2020a, p. 2, tradução nossa) ${ }^{14}$

Desse modo, percebe-se que, apesar da pandemia se expandir globalmente, seus impactos na segurança alimentar são mais intensos em alguns grupos e países específicos. Em

$14 \quad[. .$.$] devising appropriate policy measures, maintaining$ and upscaling humanitarian food security interventions, and protecting the livelihoods and food access of the most vulnerable people, particularly those in food crisis contexts. geral, maiores danos ocorrem em comunidades mais vulneráveis e que sofrem de insegurança alimentar. Países que são muito dependentes de importações de alimentos também estão em situação de vulnerabilidade .

\section{Conclusão}

Nos últimos 5 anos observa-se piora considerável na segurança alimentar mundial seja pelo aumento da fome ou pelo crescimento da obesidade (FAO et al., 2020). A pandemia de COVID-19 tende a agravar esse quadro de insegurança alimentar, principalmente nos países já afetados, caracterizando-se mundialmente como uma crise sanitária e econômica, podendo tornar-se, também, uma crise alimentar.

Além das graves consequências na saúde e na economia, a pandemia do novo Coronavírus evidenciou a fragilidade do sistema alimentar global. Salienta-se que análises feitas para 2019 indicavam que o contexto mundial para combate a fome e garantia da segurança alimentar já não era favorável. Essas condições têm piorado de forma alarmante com a pandemia e colocam sob pressão um sistema alimentar global já bastante fragilizado e carente de respostas estruturais.

As medidas que os Estados tomam para deter a disseminação do vírus têm efeitos na restrição ao comércio, reflete-se na redução dos fluxos de transportes e também na diminuição da atividade do trabalho, provida pela mão de obra assalariada. Tais medidas de restrição precisam ser acompanhadas de contrapartida econômica do Governo e até de organizações internacionais, sem as quais providências para o controle sanitário podem gerar agravamento da insegurança alimentar dos mais pobres em vários países. 
O impacto da pandemia sobre a segurança alimentar e sobre o sistema agrícola ainda não é nitidamente conhecido, uma vez que a disseminação, os efeitos e a evolução da COVID-19 se dá de diferentes formas nas diversas regiões do globo. Porém, já é possível identificar efeitos negativos sobre as pessoas ao longo da cadeia de suprimento de alimentos, ou seja, desde o pequeno produtor, os transportadores, até os consumidores finais, ressaltando as inseguranças que afetam as populações mais vulneráveis, principalmente aquelas que vivem em países que já se encontravam em crise alimentar (FAO, 2020d). Por exemplo, uma das consequências é o grande aumento do desemprego no Brasil e em outros países da ALC, com impacto importante na capacidade das famílias adquirirem os alimentos necessários à sua sobrevivência.

Essa situação de calamidade acaba exigindo dos países resposta rápida e efetiva, o que muitas vezes não tem sido observado. Particularmente no caso do Brasil, alguns elementos tornam ainda mais desafiador o enfrentamento da crise sanitária e suas repercussões na economia: evidente desacerto entre Ministério da Saúde e Presidência da República durante os dois primeiros meses da pandemia, seguido pela falta de diretrizes nacionais claras para o enfrentamento da crise; desde meados de abril até o momento, a pasta da Saúde não possui um ministro titular; não se verifica, ainda, parecer e diretrizes gerais do Ministério da Saúde para todo o país, o que gera posicionamentos divergentes entre os vários níveis de governo sobre a abordagem a ser dada à crise sanitária. Tudo isso gera dúvidas na população e mina protocolos internacionais de saúde envolvendo o isolamento social e outras medidas como, por exemplo, o uso da máscara.
Além disso, observam-se falhas importantes no amparo social aos mais vulneráveis. Milhares de pessoas ficaram desempregadas ou tiveram seus salários reduzidos e ainda assim têm enfrentado dificuldades para receber o "Auxílio Emergencial" concedido pelo governo; falhas no aplicativo destinado ao pagamento desse Auxílio, recebimentos indevidos, solicitações de auxílio negadas, comunicação insuficiente com o cidadão, dentre outras. Essas situações pressionam o sistema social como um todo e são fatores que vão delineando um cenário para degradação da segurança alimentar, dentre outras vulnerabilidades.

Ainda considerando o atual cenário, depois de quatro meses desde o início das medidas de isolamento social, o Brasil tem despertado para as consequências de não dispor de políticas específicas para a produção agrícola, o que tem resultado em redução considerável de estoques de produtos essenciais da cesta básica, como trigo, feijão, arroz, farinha de mandioca e milho. Além do possível desabastecimento desses itens essenciais, verifica-se alta de preço de produtos como verduras, legumes e frutas (SAMPAIO, 2020). Caso os Projetos de Lei que visam ajudar os agricultores e prover condições de abastecimento de alimentos não sejam votados a tempo, ou sejam inefetivos, o Brasil poderá enfrentar, além da crise política, econômica e de saúde, também uma crise alimentar.

No contexto internacional, a ONU vem atendendo vários países por meio de programas assistenciais como o Programa Mundial de Alimentos, a Iniciativa sobre o aumento dos Preços dos Alimentos, o Fundo Internacional de Desenvolvimento Agrícola (FIDA), entre outros (ONU, 20-?). Porém, devido à grave situação da pandemia em centenas de países, os doadores e financiadores internacionais podem 
priorizar ajudar seus países ao invés de continuar a ajudar um programa no exterior. Isso acaba diminuindo o financiamento desses Programas, comprometendo a capacidade de resposta humanitária internacional.

Dessa forma, a pandemia pode trazer consigo outro desafio que é a manutenção da cooperação internacional, por meio da transposição de novos reveses à superação de dilemas de ação coletiva. Tal superação demanda que os países que possuem melhores condições para responder aos desafios da segurança alimentar aumentem seus programas de proteção às pessoas em vulnerabilidade social, alimentar e de saúde, além de manter seus compromissos internacionais. Isso porque a pandemia, por sua natureza, não é um problema que afeta exclusivamente a política e as condições de respostas domésticas, mas ultrapassa fronteiras geográficas e político administrativas; ações para a sua contenção e enfrentamento são um problema global e não apenas de uma ou outra região ou país.

\section{Referências}

BALABAN, Daniel. Brasil está voltando ao mapa da fome, diz diretor da ONU. Jornal O Estado de São Paulo. Seção Entrevista. São Paulo. 12 mai. 2020. p. A9.

BEASLEY, David M. Covid-19 could detonate a 'hunger pandemic.' With millions at risk, the world must act. The Washington Post. 21 de abril de 2020. Disponível em: https://www. washingtonpost.com/opinions/2020/04/22/covid-19-could-detonate-hunger-pandemic-with-millions-risk-world-must-act/. Acesso em: 10 mai. 2020.

CEBRAFRICA. Centro Brasileiro de Estudos Africanos. União Africana. 2020. Disponível em: https://www.ufrgs.br/ cebrafrica/uniao-africana/. Acesso em: 10 mai. 2020.

CEPAL. Comisión Económica para América Latina y Caribe. Informe Especial Covid-19. n. 1. 2020a. 03 abr. 2020. Disponível em https://repositorio.cepal.org/bitstream/handle/11362/45337/6/S2000264_es.pdf. Acesso em: 12 mai. 2020.

CEPAL. Comisión Económica para América Latina y Caribe. Pandemia del COVID-19 llevará a la mayor contracción de la actividad económica en la historia de la región: caerá
$-5,3 \%$ en 2020. CEPAL, 2020b. Comunicado de Prensa. 21 abr. 2020. Disponível em https://www.cepal.org/es/comunicados/pandemia-covid-19-1levara-la-mayor-contraccion-la-actividad-economica-la-historia-la. Acesso em: 12 mai. 2020.

CULLEN, Maximo Torero. Coronavirus Food Supply chain under Strain what to do. 2020a. Food and Agriculture Organization of the United Nations. 24 mar. 2020.

CULLEN, Maximo Torero. COVID-19 and the risk to food supply chain: How to respond? 2020b. Food and Agriculture Organization of the United Nations. 29 mar. 2020. Disponível em: http://www.fao.org/3/ca8388en/CA8388EN.pdf. Acesso em: 08 mai. 2020.

FAO. Organização das Nações Unidas para a Alimentação e a Agricultura. The State Of Food And Agriculture. Roma. 1996. Disponível em: http://www.fao.org/docrep/003/ w1358e/w1358e00.htm . Acesso em: 10 mai. 2020.

FAO. Organização das Nações Unidas para a Alimentação e a Agricultura. E-learning Centre. Food Security Concepts and Frameworks. 2008. Disponível em: http://www.fao.org/ elearning/\#/elc/en/Course/FC. Acesso em: 10 mai. 2020.

FAO. Organização das Nações Unidas para a Alimentação e a Agricultura. Ebola Strategy: Ebola and Marburg virus disease epidemics: preparedness, alert, control and evaluation. August, 2014a. Disponível em https://apps.who.int/iris/bitstream/ handle/10665/130160/WHO_HSE_PED_CED_2014.05_ eng.pdf; jsessionid=4295767ABBDE8A7376FED6966CB9A36D? sequence $=1$. Acesso em: 11 mai. 2020.

FAO. Organização das Nações Unidas para a Alimentação e a Agricultura. The State of Food Insecurity in the World. 2014b. Disponível em: http://www.fao.org/3/a-i4030e.pdf. Acesso em: 22 out. 2017.

FAO. Organização das Nações Unidas para a Alimentação e a Agricultura. Panorama de la seguridad alimentaria y nutricional: desigualdad e sistemas alimentarios. Organización de las Naciones Unidas para la Alimentación y la Agricultura (FAO); Organización Panamericana de la Salud (OPS); Programa Mundial de Alimentos (WFP); Fondo de las Naciones Unidas para la Infancia (UNICEF) Santiago, 2018. Disponível em http://www.fao.org/3/ca2127es/ca2127es.pdf. Acesso em: 11 maio 2020.

FAO. Organização das Nações Unidas para a Alimentação e a Agricultura. The State of Food Security in the World: Safeguarding against economic slowdowns and downturns. 2019. Disponível em: http://www.fao.org/3/ca5162en/ca5162en.pdf . Acesso em: 07 mai. 2020.

FAO. Organização das Nações Unidas para a Alimentação e a Agricultura. Anticipating the impacts of COVID-19 in humanitarian and food crisis contexts. 2020a. Disponível em: http://www.fao.org/3/ca8464en/CA8464EN.pdf . Acesso em: 8 abr. 2020.

FAO. Organização das Nações Unidas para a Alimentação e a Agricultura. COVID-19 pandemic - impact on food and 
agriculture. 2020b. Novel Coronavirus (COVID-19). Disponível em: http://www.fao.org/2019-ncov/q-and-a/impact-on-food-and-agriculture/en/. Acesso em: 13 maio 2020.

FAO. Organização das Nações Unidas para a Alimentação e a Agricultura. World Food Situation. Fao Food Price Index. 2020c. Disponível em: http://www.fao.org/worldfoodsituation/foodpricesindex/en/. Acesso em: 08 abr. 2020.

FAO. Organização das Nações Unidas para a Alimentação e a Agricultura. 25 países de América Latina e do Caribe se coordenam para apoiar o funcionamento regular do sistema alimentar durante a crise da COVID-19. FAO Brasil, 2020d. Notícias. 03 abr. 2020. Disponível em http://www.fao.org/brasil/ noticias/detail-events/pt/c/1269594/. Acesso em: 12 maio 2020.

FAO. Organização das Nações Unidas para a Alimentação e a Agricultura. Coronavirus disease 2019 (COVID-19) - Addressing the impacts of COVID-19 in food crises. 2020e. Disponível em: http://www.fao.org/emergencies/appeals/detail/en/c/1270012/. Acesso em: 06 mai. 2020.

FAO. Organização das Nações Unidas para a Alimentação e a Agricultura. La nueva enfermedad coronavirus (COVID-19) y los Sistemas Alimentarios en América Latina y el Caribe. 23 jul 2020. 1 vídeo (01h16min) Publicado por Food and Agriculture Organization on the United Nations. 2020f. Disponível em: http://www.fao.org/americas/publicaciones-audio-video/ covid19-y-sistemas-alimentarios/es/. Acesso em: 28 jul. 2020.
FAO et al.The State of Food Security and Nutrition in the World 2020. Transforming food systems for affordable healthy diets. Rome, FAO. 2020. Disponível em: http://www.fao. org/3/ca9692en/CA9692EN.pdf . Acesso em: 25 jul. 2020.

FSIN. Food Security Information Network. 2020 Global Report On Food Crises: Joint Analysis For Better Decisions. 2020. Disponível em: https://www.fsinplatform.org/sites/default/files/resources/files/GRFC_2020_ONLINE_200420. pdf . Acesso em: 07 mai. 2020.

ONU. Organizações das Nações Unidas. A Onu e a alimentação. 20-?. Disponível em: https://nacoesunidas.org/acao/ alimentacao/ Acesso em: 27 jun. 2020.

ONU NEWS. Organizações das Nações Unidas. Ministros africanos e FAO fazem reunião virtual para enfrentar covid-19. 2020. Disponível em: https://news.un.org/pt/ story/2020/04/1710552. Acesso em: 06 mai. 2020.

SAMPAIO, Cristiane. Estoque de alimentos está abaixo dos $20 \%$ necessários ao país, alerta especialista. Brasil de Fato. 24 jun. 2020. Disponível em: https://www.brasildefato.com. br/2020/06/24/estoque-de-alimentos-esta-abaixo-dos-20-necessarios-ao-pais-alerta-especialista Acesso em: 27 jun. 2020.

WORLDOMETERS. Coronavírus. [20-?] Disponível em: https://www.worldometers.info/coronavirus/. Acesso em: 13 maio 2020 . 